\title{
Relación entre el cambio de uso del suelo en la cuenca del Aconcagua y su litoral arenoso correlativo. Chile central ${ }^{1}$
}

\author{
María Victoria Soto Bäuerle², Joselyn Arriagada González², \\ Carmen Paz Castro Correa², Michael Maerker ${ }^{3}$ \\ y Giuliano Rodolfi ${ }^{4}$
}

\begin{abstract}
RESUMEN
En la cuenca del Aconcagua los procesos de modernización e intensificación del uso de suelo se han traducido tanto en una reconversión agrícola como en el aumento de la superficie explotada. Tal proceso ha sido muy intenso en la medida que se han incorporado nuevos terrenos, sobrepasando los límites del valle y orientándose hacia tierras en pendiente. Ello ha incidido en los procesos de transferencia de masa desde la cuenca hacia la desembocadura. Para ello, se evidencian cambios de uso del suelo a través de los modelos de cobertura vegetacional; el análisis de la geomorfología de detalle en el litoral da cuenta de la ocurrencia de transformaciones en la playa y las dunas, que son territorialmente poco significativas, pero evolutivamente importantes, toda vez que evidencian una sobre alimentación de sedimentos, que podría estar correlacionada con los cambios ocurridos en la cuenca.
\end{abstract}

Palabras clave: Cambio de uso del suelo, aporte sedimentario, dunas.

\begin{abstract}
In the Aconcagua basin the modernization and intensification of land use have resulted both in an agricultural conversion, and the increase in area cultivated. This process has been very intense as far that have entered new areas beyond the limits of the valley and towards sloping ground. This signals changes in land use over the vegetation coverage models. The analysis of the geomorphology of the coastline in detail, accounts for the occurrence of transformations on the beach and dunes that are spatially less significant, but evolutionarily important, since they show a sediment feeding, which could be correlated with changes in vegetation cover in the basin
\end{abstract}

Key words: Land use change, sediment input, coastal sand dunes.

1 Proyecto FONDECYT No 1071098. Artículo recibido el 23 de marzo de 2010, aceptado el 30 de junio de 2011 y corregido el 21 de octubre de 2011.

2 Departamento de Geografía, Universidad de Chile (Chile).E-mail:mvsoto@uchilefau.cl; joarriag@uchile.cl; cpcastro@uchilefau.cl
3 Institut für Geoökologie, Universität Potsdam (Alemania). E-mail: michael.maerker@geographie.uni-tuebingen.de 4 Universidad de Florencia (Italia). E-mail: giuliano.rodolfi@unifi.it 
En la actualidad, entre los diversos procesos espaciales derivados de la globalización de la economía, uno de los aspectos que tiene una alta incidencia en la organización espacial dice relación con la creciente valorización de aquellos territorios, cuyos recursos y productos tienen una alta demanda en el mercado mundial. Chile no es una excepción a esta dinámica ya que desde mediados de la década de los años 70 del siglo recién pasado, el modelo económico ha cambiado en forma decisiva.

En este escenario, los intensos y crecientes procesos productivos agrícolas desarrollados en el valle del Aconcagua, han implicado la expansión territorial de los cultivos de frutales más allá de los suelos aluviales del fondo de valle, ocupando terrenos de conos, glacis y laderas, con una consecuente intervención en la dinámica del paisaje natural.

Los impactos generados en el paisaje asociados a los diferentes niveles de intervención por la expansión y modernización agrícola, constituyen temas de importancia global. En ambientes mediterráneos de Italia, Rodolfi (1988) demostró el impacto de la agricultura desde la postguerra y sus efectos en la generación de condiciones de inestabilidad geomorfológica. Pelacani et al. (2006a, 2006b) a su vez, enfatiza la importancia del análisis del suelo y de la erodabilidad en ambientes con alto impacto antrópico, especialmente vinculados a las actuales prácticas de manejo del suelo. Los autores dan cuenta que las alteraciones topográficas, principalmente aquellas realizadas en las laderas, generan condiciones de susceptibilidad a movimientos en masa y erosión del suelo, tanto actuales como de data histórica.

Los territorios más vulnerables a tales intervenciones son aquellos en que el clima se caracteriza por la escasez o bien la irregularidad de las precipitaciones, las cuales suelen ser esporádicas e intensas, por lo que aceleran considerablemente la erosión del suelo (Rodolfi, 2006). En este contexto, las relaciones existentes entre el cambio climático y erosión del suelo, ha sido documentada para el ambiente mediterráneo por Rodolfi y Sanchi (2002), en el sentido que los procesos de degradación y desertificación son atribuidos a las actividades humanas, pero exacerbados por las condiciones climáticas, sobre todo en áreas sujetas a una rápida y progresiva intensificación de la agricultura, además de ser esta muy mecanizada y tecnificada.

En el contexto anterior, los aportes de Castro et al. (2010); Aliaga y Leyton (2008); Meza (2010) para la cuenca del Aconcagua, dan cuenta de que existen impactos negativos en la geodinámica actual del paisaje debido a la reconversión productiva y a la pérdida de suelo. Así, se observa una estrecha relación entre la configuración espacial del grado de erosionabilidad de los suelos y la pendiente del terreno, donde los altos niveles de erosión están asociados a superficies desprovistas de vegetación, y los niveles moderados, a bosques, suelos agrícolas, matorrales y praderas.

Desde el punto de vista del manejo, la preparación del terreno para la agricultura en pendiente, implica fuertes alteraciones en la topografía, la cobertura vegetal, estabilidad del sustrato y pérdida de suelo (Figura $N^{\circ} 1$ ).

Si tal situación es analizada, además, en el escenario de los cambios climáticos y de las fluctuaciones de los eventos El Niño, se debe esperar un significativo incremento de los procesos dinámicos en el paisaje. Según los antecedentes de modelización climática de Garreaux et al. (2008), en Chile central $\left(31^{\circ}-37^{\circ}\right)$ habría una pérdida generalizada de precipitaciones con excepción de la estación de otoño para latitudes inferiores a $33^{\circ}$. La pérdida es del orden $40 \%$ en las tierras bajas ganando magnitud hacia la ladera andina durante el verano, pero reduciéndose durante el otoño y el invierno. También se alude al impacto hidrológico en la medida que habría una reducción del área andina capaz de almacenar nieve y considerando que la isoterma $0^{\circ}$ sufriría un alza de altura por el proceso de calentamiento, las crecidas fluviales invernales se verían incrementadas por el aumento del área de las cuencas aportantes.

Al analizar la cuenca desde el punto de vista de las transformaciones productivas, los impactos derivados deben ser considerados sistémicamente tanto en la cuenca como en su litoral adyacente, tal como lo señalan los tratados actuales de gestión de costas y de cuencas. Ello está referido a la relación coordinada y planificada de localización de usos 
ambientales, socioculturales y sustentables en las cuencas y sus litorales correlativos (Comisión Europea 2002; Romay y Maceira 2006; Bachmann et al., 2007).

El impacto asociado a tales transformaciones se debiera traducir en la generación de condiciones favorables para el aumento del aporte de masa desde los sistemas morfológicos intervenidos, hacia la red de drenaje asociada. De acuerdo con los modelos teóricos tradicionales y actuales, los sistemas fluviales constituyen la mayor fuente aportante de sedimentos a los océanos y los ambientes marinos y costeros son los receptores de la mayoría de los sistemas fluviales. Existe en consecuencia una fuerte conexión entre los procesos de erosión o depositación que ocurren en la cuenca y cómo estos se ven reflejados en el aporte sedimentario de los ambientes marinos y costeros (Stallery y Phillips 2011).

Ejemplo de ello son los grandes ríos del mundo, como el Mississippi, gran generador de aportes sedimentario depositado en su delta correlativo, cuya cuenca ha sufrido transformaciones relacionales con el uso del suelo de praderas a un uso de agricultura intensiva
(Santschi et al., 2007) aumentando consecuentemente su carga sedimentaria. Esta dinámica ha sido propia de los grandes ríos en las últimas décadas, la alteración de los ríos por las actividades humanas y el aumento del flujo sedimentario al océano por la agricultura intensiva y la deforestación; también lo ha sido la disminución de la carga debido a la construcción de embalses y represas (Wang et al., 2010).

Las tendencia futuras dan cuenta que el flujo sedimentario desde las cuencas hidrográficas hacia las zonas costeras debido a la acción humana y el cambio climático serán una constante (Restrepo et al., 2006). Ello fue hipotetizado también para la cuenca del río Choapa (semiárido de Chile), por Arriagada (2009) y Soto et al. (2010).

Otra consecuencia de la intervención en las cuencas, es la reducción de sedimentos y el aumento de la erosión costera, situación que genera serias implicancias desde el punto de vista de la valorización económica de las zonas costera y el impacto en el poblamiento de las mismas; estos hechos, Panda y Mohanthy (2011) los atribuyen a una señal de la declinación de la carga sedimentaria, atribuible

Figura $\mathrm{N}^{\circ} 1$

Preparación del terreno para agricultura en pendiente

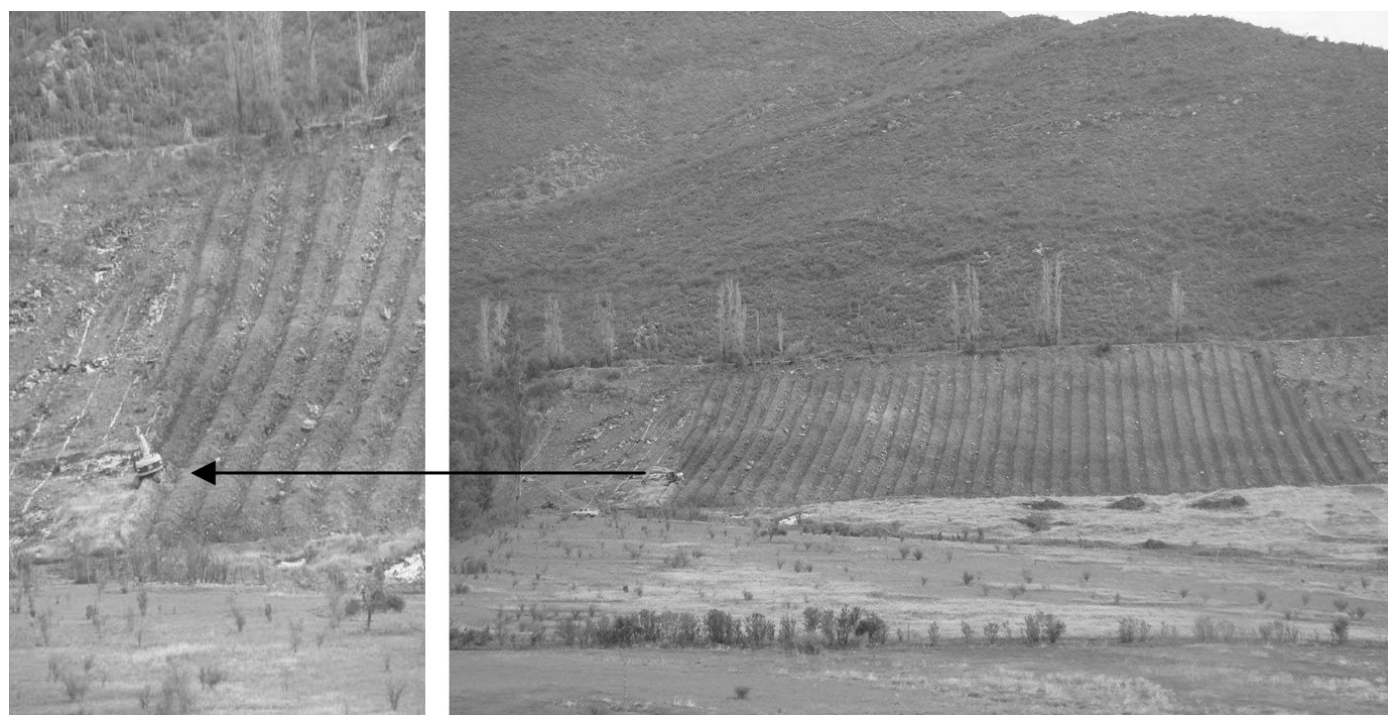

Fuente: Elaboración propia. 
a la variabililidad de los cambios climáticos recientes y su interacción con los actividades antrópicas.

En este contexto, y desde una perspectiva sistémica, los impactos de los procesos de intervención en la cuenca debieran verse reflejados a través de determinadas manifestaciones morfológicas, tanto en la sección de montaña y valle de la cuenca, como en el sistema litoral correlativo. Sin embargo la conexión entre cambios en la dinámica sedimentaria con el sistema fluvial y la carga de masa a la desembocadura de los ríos no siempre es fuerte o directa (Stallery y Phillips 2011).

El sistema estudiado está conformado por un complejo deltaico, un extenso litoral arenoso y el campo de dunas asociado de Ritoque. La zona litoral de la cuenca, morfológicamente corresponde a un sistema de desembocadura clasificado como estuario dominado por procesos fluviales y por el oleaje (Cortez, 2002; Martínez \& Cortez 2007). El litoral asociado presenta un gran campo de dunas de diferente generación, las dunas estabilizadas pleistocénicas (Paskkof 1970) y las dunas actuales, correspondientes a un complejo de dunas anteriores naturales (nebkas), artificiales y el extenso campo de dunas que Castro (1987) clasificó como transversales y barjanoides.

De acuerdo a los antecedentes anteriores, surge el propósito de esta investigación, que es analizar la relación entre el sistema natural costero y los cambios de uso del suelo que se están desarrollado en el valle del Aconcagua. Esta pregunta de investigación se analiza a través de la respuesta sedimentaria en el litoral arenoso correlativo, toda vez que se parte de la hipótesis que debido a los cambios de uso del suelo y a las prácticas de manejo del suelo para la agricultura de frutales, tanto en el fondo de valle como en ladera, se debiera observar un aumento en el abastecimiento de masa en playas y dunas del litoral de Ritoque.

\section{Área de estudio}

El valle del Aconcagua, en la Región de Valparaíso, corresponde a una cuenca transversal, de condiciones climáticas templadas mediterráneas. La presencia de numerosas y amplias subcuencas y valles intermontanos de media y baja montaña, y el fondo de la depresión, ha permitido la formación de un suelo de excelente aptitud agrícola, configurando históricamente a la cuenca con una clara vocación productiva agrícola.

El área de estudio comprende las áreas de media y baja montaña andina y costera, los valles aluviales, las formas de contacto de ladera/valle (conos y glacis), que han sido incorporados a la agricultura, considerando un período de 35 años. La zona del exutorio fue analizada como la componente que cumple la función morfodinámica de ser receptora de masa desde la cuenca y comprende al estuario, la playa y las dunas de Ritoque (Fig. 2).

\section{Materiales y métodos}

El valle del Aconcagua fue analizado como un sistema territorial integrado, focalizado en el cambio de uso y manejo del suelo y la ocupación de terrenos sin aptitud agrícola para estos fines, con una tendencia marcada hacia la agricultura intensiva de exportación, propia de la economía globalizada del país. A partir del punto de vista de que las prácticas agrícolas repercuten en fuertes alteraciones al paisaje natural, con remoción de la cobertura vegetal y de suelo, en un paisaje a ser caracterizado por el incremento de las precipitaciones concentradas, que representarían las condiciones actuales y futuras para la generación transporte y depositación de una sobrecarga de masa desde la cuenca a la línea de costa. Las componentes del sistema territorial consideradas, se expresan en la Figura $N^{\circ} 3$.

\section{Procesos indicativos de cambio en}

\section{la cuenca}

Uno de los aspectos relevantes ha sido el cambio de uso del suelo en la cuenca. A partir de los estudios realizados por Aliaga y Leyton (2008), Castro et al. (2010) y Meza (2010), referidos a tales cambios, a las prácticas de manejo y a los impactos asociados. Se estableció como condición de base un estado de cambio que debía ser analizado para la cuenca en su totalidad. La verificación en terreno de evidencias de erosión y de aporte de sedimentos desde los terrenos labrados hacia los cursos de agua, también sirvió como elemento de ratificación de la denominada condición de cambio en la cuenca por razones antrópicas. 
Figura $\mathrm{N}^{\circ} 2$

Área de estudio

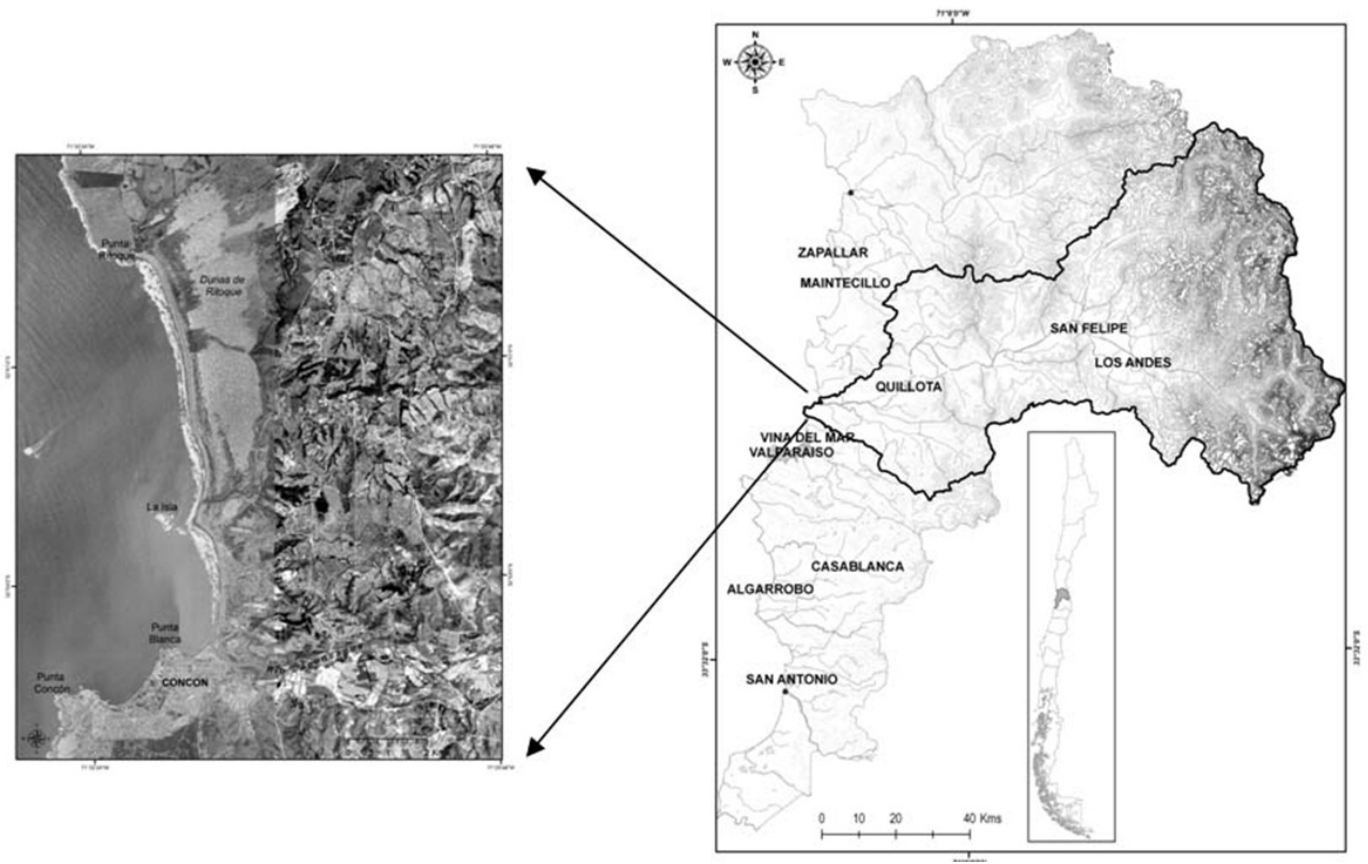

Fuente: Elaboración propia.

Figura $\mathrm{N}^{\circ} 3$

Esquema metodológico de la investigación

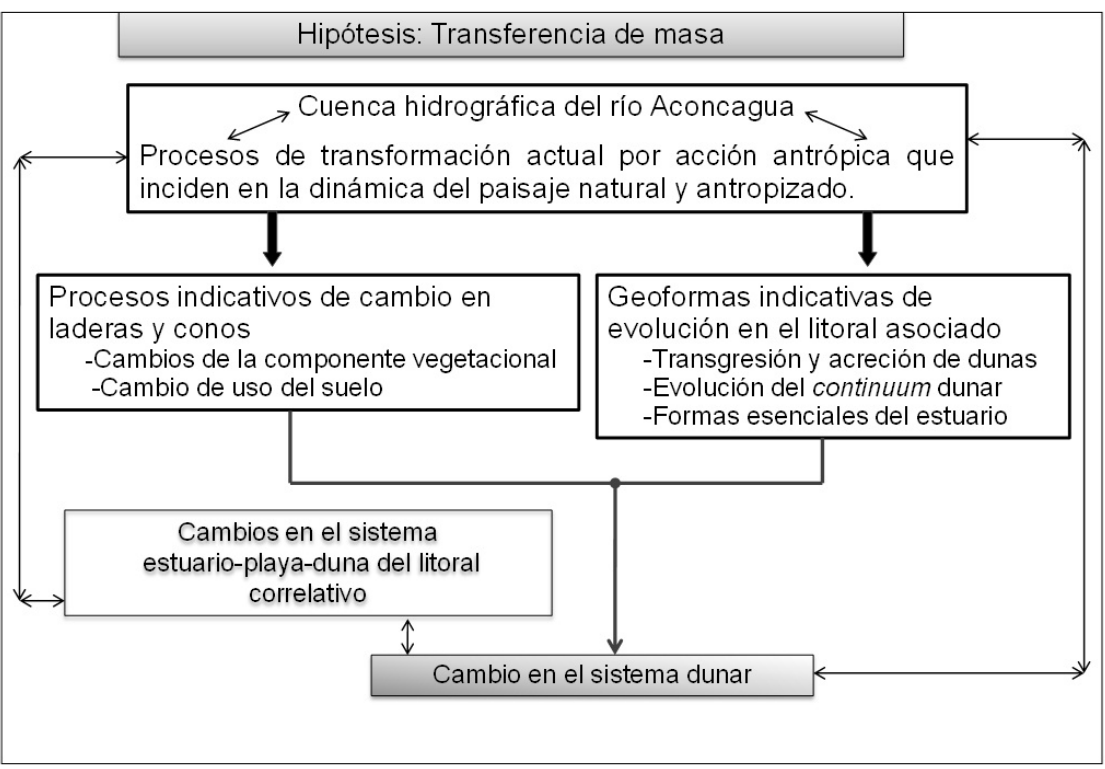

Fuente: Elaboración propia. 
Se establecieron los patrones de cambio vegetacional para un período de 35 años, en función de la disponibilidad de imágenes, a fin de aplicar el indicador NDVI. Se emplearon imágenes LANDSAT 7 ETM con una resolución de $30 \mathrm{~m} \times 30 \mathrm{~m}$ para los años 1989, 1999 y 2004. El NDVI (Normal Difference Vegetal Index) se obtuvo mediante procesamiento SIG con software IDRISI ANDES y ArcMap. Se emplearon los criterios de reclasificación de Marker et al. (2001), cuyos rangos de cobertura vegetal (Cuadro $\mathrm{N}^{\circ} 1$ ) fueron validados para Chile por Fernández (2006).

\section{Cuadro $\mathrm{N}^{\circ} 1$}

Rangos de cobertura vegetal

\begin{tabular}{|l|c|}
\hline \multicolumn{1}{|c|}{ Cobertura vegetal } & $\%$ \\
\hline Muy baja & $0-10$ \\
Baja & $10-25$ \\
Moderadamente baja & $25-50$ \\
Moderadamente alta & $50-75$ \\
Alta & $75-90$ \\
Muy alta & $90-100$ \\
\hline
\end{tabular}

Fuente: Marker et al. (2001).

Este indicador fue aplicado tanto en la cuenca como en el campo de dunas. En el primer caso para dar cuenta de los cambio vegetacionales ocurridos, y en la dunas, específicamente buscando la tendencia a la disminución de la vegetación, lo que es asociable hipotéticamente a la acreción y transgresión de dunas, tanto al interior del sistema o hacia el exterior del campo dunar.

\section{Geoformas litorales asociadas a la cuenca}

Se analizaron los patrones de cambio y tendencia evolutiva de la morfología aledaña a la desembocadura, a través de la recopilación de información, fotointerpretación y trabajo de terreno. Junto con analizar las formas esenciales del sistema de desembocadura, se hizo énfasis en las formas de la playa, sobre todo de las dunas anteriores y la dinámica de los sistemas dunares.

Desembocadura: el análisis de la desembocadura del Aconcagua se basó en los resultados de Cortez (2002), quien determinó que la tendencia evolutiva observada entre 1877 y 1996, refleja importantes variaciones morfológicas, siendo la más evidente la sedimentación progresiva del sector de la desembocadura. Ello se expresa en el crecimiento de las barras litorales y su incidencia en los bancos medios estuariales. Los bordes de estos bancos son convexos como respuesta a una tendencia deposicional. Por otra parte, las flechas litorales y los bancos de la zona interior y media, son los elementos que presentan la mayor estabilidad en el periodo analizado.

Se consideró como base conceptual, la sistematización de Araya Vergara (1981), que identifica como formas esenciales los meandros y bancos estuariales, bancos laterales cuspidados, laguna estuarial y flecha litoral, localizados en las zonas proximales, medias y distales, respectivamente:

Cuadro $\mathrm{N}^{\circ} 2$

Sistematización de Araya Vergara (1981)

\begin{tabular}{|c|c|c|c|c|}
\hline & Zona distal & Zona media & Proximal & \\
\hline \multirow{2}{*}{ Influencia marina } & Flecha litoral & \multirow{2}{*}{$\begin{array}{l}\text { Bancos medios } \\
\text { estuariales }\end{array}$} & \multirow{2}{*}{$\begin{array}{l}\text { Meandros } \\
\text { estuariales }\end{array}$} & \multirow{2}{*}{ Influencia fluvial } \\
\hline & Laguna estuarial & & & \\
\hline
\end{tabular}

Fuente: Araya Vergara, 1981. 
Se realizó la identificación de las formas citadas a través de fotointerpretación de fotografías aéreas de un lapso de 35 años, así como también del reconocimiento en terreno. Se utlilizaron herramientas SIG, del software ArcGis 9.2.

Playa arenosa: se aplicó el concepto de playa como receptora de la carga sedimentaria transportada por las corrientes de playa (Massenlik et al. 2006), en donde además se expresan las condiciones hidrodinámicas del litoral. En este contexto, la presencia y aumento de cobertura de formas depositacionales en la playa arenosa, como microdunas y dunas anteriores se consideró como una condición de aporte sedimentario. De la misma manera, el tipo de playa dominada por olas de Wrigth \& Short (1984) en Short (1999), fue visto como el escenario hidrodinámico de transferencia de masa correlativo a la cuenca hidrográfica.

Dunas: la zona costera es analizada a través de fotos aéreas en diferentes años, en un estudio evolutivo las formas de las dunas, con sustento en el concepto de continuum dunar. El principal método de análisis se refiere al principio cartográfico de seguimiento del diseño de las crestas dunares, las cuales se clasificaron de acuerdo a las nociones de secuencia (Verstappen, 1972) y de continuum (Araya-Vergara, 1986 y 1987), combinándose para determinar las familias de dunas (Fig. 4), sean estas derivadas de dunas anteriores o de barjanes. La relación entre las dunas de la playa arenosa y la evolución de las dunas interiores libres, se relacionó a fin de establecer la tendencia de cambio del sistema dunar.

\section{Resultados}

\section{Cambio de la cobertura vegetal y expansión de la frontera agrícola}

El valle del Aconcagua se caracteriza por su clara vocación productiva vinculada a la agricultura, la cual ha presentado una clara tendencia a un aumento de la superficie ocupada especialmente en la producción de paltos, en las últimas décadas. El área de la cuenca destinada al uso agrícola equivale al $12 \%$ de la superficie total, con una producción del $41 \%$ del total de paltos del país, de plantaciones en terrenos con fuerte pendiente y prácticas de manejo rentables a la producción, principalmente de exportación, han provocado un aumento de erosión y de pérdida de vegetación natural (Aliaga y Leyton, 2008).

A partir de los antecedentes aportados para las comunas de Quillota, Concón y Los Andes por Castro et al. (2010) y Meza (2010) en Quillota, en términos de los impactos de las actividades agrícolas en el suelo, y a fin de establecer los cambio de uso ocurridos en el período de 40 años, se utilizó el indicador de cobertura vegetal, NDVI. Los resultados arrojados por ese tipo de procedimientos dan cuenta de un progresivo aumento de la cobertura vegetal (Fig. 5), siendo importante destacar al respecto, que tales cambios deben ser analizados a la luz de la escala de análisis, la resolución de las imágenes y las fechas de las mismas.

Figura $\mathrm{N}^{\circ} 4$

Familia de dunas y continuum dunar

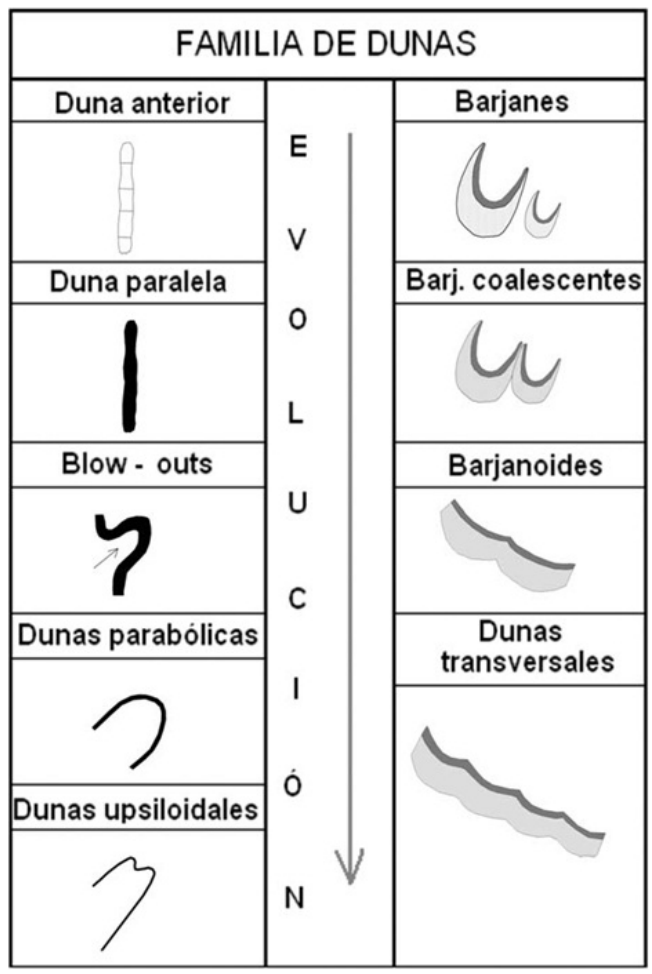

Fuente: Arriagada, 2009. 
No obstante lo anterior, al observar la configuración de las coberturas de vegetación en los años, 1975, 1989 y 2006, correspondientes al mes de marzo, es posible aseverar una tendencia permanente a la expansión espacial de las plantaciones, con una densificación de la cobertura. Destaca el proceso ocurrido en el valle, en las comunas de San Felipe y Los Andes, en términos del patrón de expansión y densificación de la cobertura vegetacional, longitudinalmente y hacia las laderas de la cuenca, entre los años 1989 y 2006 (Figura N 5).

Figura $N^{\circ} 5$

Resultados de NDVI para la cuenca del Aconcagua
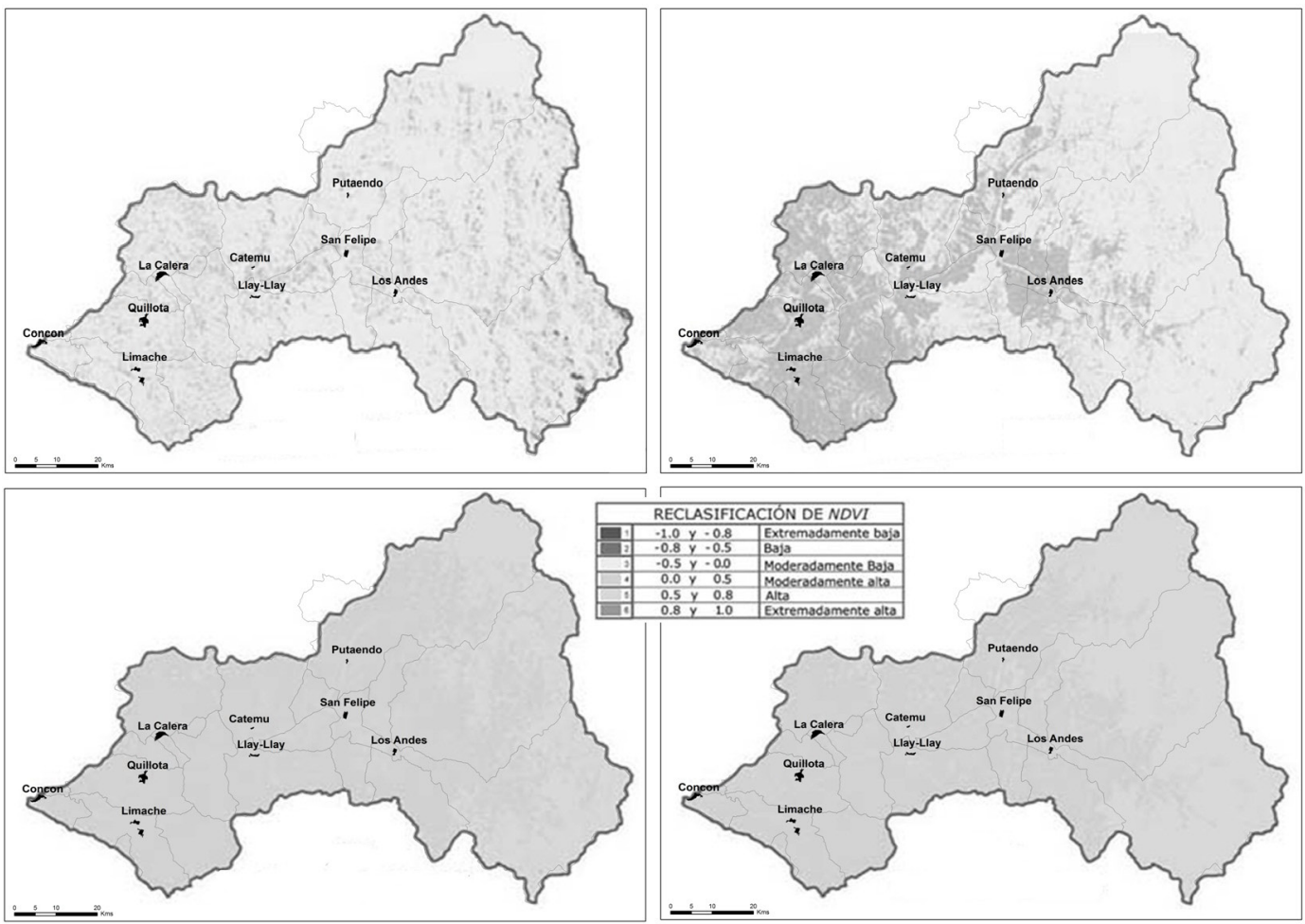

ECIASIFICACTÓN DE NDVI

\begin{tabular}{|l|l|}
\hline$-1.0 y-0.8$ & Extremadamente baja \\
\hline $.0 .8 y-0.5$ & Baja \\
\hline $.0 .5 y-0.0$ & Moderadamente $8 . j 5$
\end{tabular}

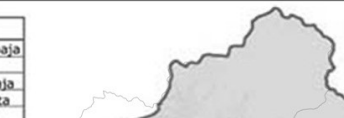

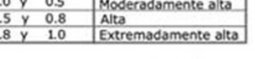

Fuente: Proyecto Fondecyt 1071098.

De la misma manera, a partir del supuesto que los procesos vinculados a los cambios de uso y su impacto en la erosión del suelo, debieran redundar en un aumento de la masa sedimentaria recibida en el litoral, se analizaron las condiciones de cambio vegetacional en el campo de dunas de Ritoque. Sin embargo, a partir de los resultados del NDVI, esta relación no es notoria, pues no se observa una disminución de vegetación (Figura $N^{\circ}$ 6). En el contexto de las precauciones al analizar los resultados del indicador NDVI ya señaladas, es posible no obstante, constatar condiciones de cambio, las que han estado claramente orientadas al aumento de la cobertura vegetacional, tal como se observa en la zona palustre y en el balneario de Ritoque, que pueden estar muy vinculadas a las fechas de las imágenes, como al nivel general del sistema depositacional. A esa escala, se puede establecer que ha habido un aumento de la vegetación como parte del crecimiento y expansión natural de las especies introducidas a partir de la década de 1970, asociadas a los planes de control y manejo de dunas. 
Figura $\mathrm{N}^{\circ} 6$

NDVI de la sección costera de la cuenca del Aconcagua. Campo de dunas de Ritoque
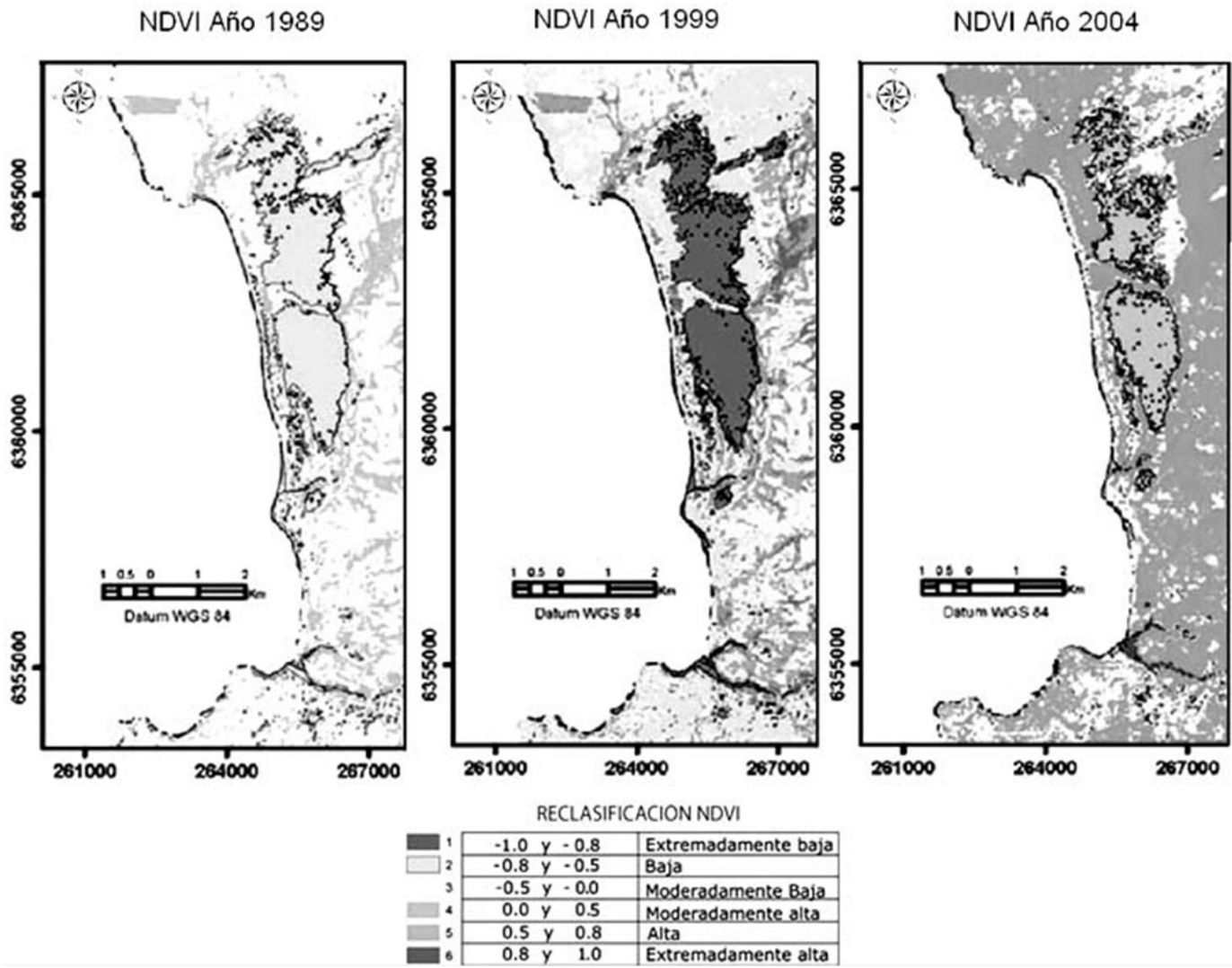

Fuente: Soto et al., 2009.

No obstante lo anterior, la verificación en terreno da cuenta de dos situaciones diferentes pero complementarias y propias de la dinámica del sistema. Una es la casi total estabilización del margen interno del campo de dunas de Ritoque, que se presenta conformado por un frente de transgresión de gran altura, pero muy controlado por la vegetación. La otra, corresponde a la ribera norte del estero Mantagua, donde se han observado evidencias de transformaciones dunarias y una marcada reactivación del frente transgresivo. Este hecho se demuestra gracias al análisis de fotos aéreas del año 1960 y observaciones en terreno del estado actual, como por ejemplo, en zonas del estero Mantagua que están siendo prácticamente sepultadas por el frente de transgresión ( $\mathrm{Fi}$ gura $\left.\mathrm{N}^{\circ} 7\right)$.
Figura $\mathrm{N}^{\circ} 7$

Estero Mantagua. Meandro siendo obstruido por aporte de arenas y frente de transgresión de las dunas de Ritoque

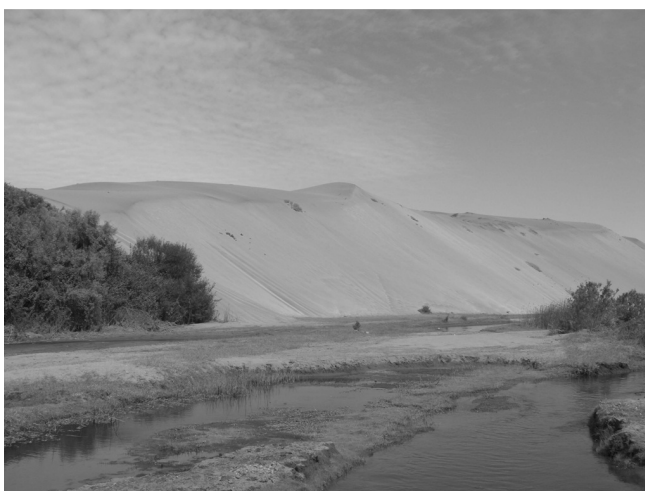

Fuente: Colección personal de los autores. 
Al constatar desde el punto de vista del uso del suelo los cambios en la cuenca y en la costa, Aliaga y Leyton (2008) estimaron la erosionabilidad en tres comunas de la cuenca (Tabla 2), datos que contribuyen a la comprensión de los procesos de transferencia de sedimentos desde la cuenca hacia el litoral y los procesos antes descritos y vinculados a los cambios de uso del suelo. A este respecto es importante destacar los valores asociados a la comuna de Los Andes, cuyo alto porcentaje estaría asociado a áreas de vasta tradición frutícola, donde se iniciaron los procesos de ampliación de las fronteras agrícolas a nivel de la cuenca. Quillota por su parte, presenta una condición de erosionabilidad en niveles medios en casi el $90 \%$ de la superficie y que debiera ser correlacionado también con la intensa proliferación de los cultivos en laderas, más allá de los tradicionales en el fondo de valle.

\section{Cuadro $\mathrm{N}^{\circ} 2$}

Superficie en porcentaje por comuna, según grado de erosionabilidad causada por el uso de suelo

\begin{tabular}{|l|c|c|c|c|c|}
\hline \multirow{2}{*}{ Comuna } & \multicolumn{5}{|c|}{ Superficie (\%) } \\
\cline { 2 - 6 } & Bajo & Medio Bajo & Medio & Medio Alto & Alto \\
\hline Concón & 13,2 & 67,6 & 6,2 & 12,1 & 0,7 \\
Quillota & 9,3 & 59,7 & 28,6 & 2,3 & 0,0 \\
Los Andes & 0,0 & 14,0 & 2,7 & 0,7 & 82,5 \\
\hline
\end{tabular}

\section{Evidencias geomorfológicas del aporte actual de masa sedimentaria a la línea de costa}

El análisis de las relaciones entre la zona de desembocadura, la playa y el campo de dunas, a través de la identificación de las geoformas existentes y las transformaciones ocurridas, constituyen evidencias de cambios en las formas de playa y dunas. En este sentido, el complejo de desembocadura del río Aconcagua presenta las formas esenciales que lo conforman, sean meandros y bancos estuariales, bancos laterales cuspidados, laguna estuarial y flecha litoral, con modificaciones que más bien pueden ser asociadas a los cambios de caudal y masa de carácter estacional, no siendo posible evidenciar a través de estas una condición evolutiva del sistema y las formas asociadas.

Lo observado en terreno durante noviembre de 2009, da cuenta por otro lado, de la importante cantidad de material detrítico llegado a la desembocadura y de la formación y mantenimiento de cordones de rodados frescos en la playa, con un perfil de playa de morfología compleja, con más de una secuencias de ante-postplaya, lo que es indicativo de la condición de desarrollo progradacional de la playa en ese momento (Fig. 8); los rodados no fueron observados hacia el sector medio y distal de la ensenada.

\section{Figura $\mathrm{N}^{\circ} 8$}

Desembocadura río Aconcagua, playa de rodados, con diferentes secuencias de playas

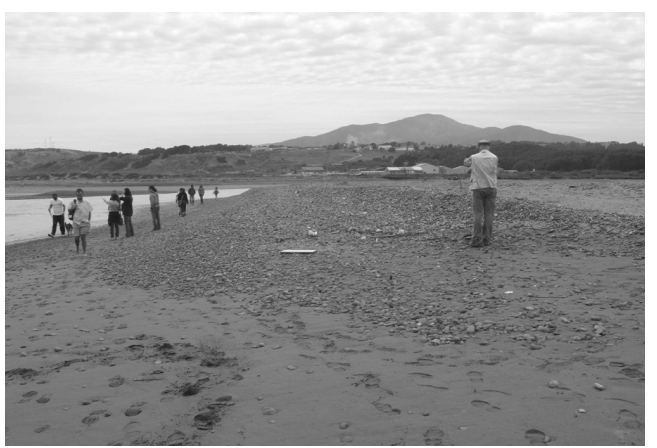

Fuente: Colección personal de los autores, noviembre de 2009. 
En relación a lo analizado en la playa arenosa de la ensenada y su campo de dunas asociado, en un análisis a escala muy local, es posible hallar claras evidencias de aporte reciente y abundante de arenas, que difieren en posición dentro del sistema, como en términos del continnum dunario. En la sección media de la ensenada, en el estero Mantagua, se encuentra el hasta ahora único frente transgresivo del campo de dunas. Su identificación estuvo facilitada por la acción de las arenas en un meandro del estero, que está siendo notoriamente sepultado (Fig. 7), y en donde el muy escaso nivel de cobertura vegetal indica tanto la permanente alimentación de arenas, como que este cambio es relativamente reciente, si se compara con las imágenes aéreas de 1960. Este sector, en el año 1960, presentaba dunas libres y sin manejo, con notorios cordones de dunas transversales de onda larga y con algunos rasgos barjanoides. En la actualidad, se evidencia la acción erosiva del viento, con el desarrollo de profundos y amplios blowout, cuyas arenas han avanzado hacia barlovento sepultando la vegetación de bosque preexistente y derramando finalmente hacia el estero.

Otro hecho morfodinámico diagnóstico de la alimentación local, es la presencia actual en la altaplaya, de dunas barjanes, aisladas y de pequeño tamaño, que se han desarrollado en un sector que hasta el año 2008 presentaba dunas anteriores del tipo nebkas, patrón que aún se presenta al norte y sur de estos nuevos barjanes. Las dunas anteriores del tipo nebkas, se encuentran estabilizadas por vegetación, son muy altas y presentan una marcada erosión de embestida del oleaje.

La existencia de estos barjanes es un indicador de una mayor alimentación de arenas y de una evolución en la familia de los barjanes a las dunas transversales (Fig. 9), no obstante anteriormente había una evolución en el sistema de la familia de dunas anteriores.

En la sección distal de la ensenada, en el borde de sotavento cercano al balneario de Ritoque, se observan nebkas y además dunas embrionarias. Desde el punto de vista de los cambios recientes, destaca el avance y crecimiento de estas nebkas en el período comprendido entre 2006-2009 (observación en terreno), en la medida que se han desarrollado y progradado hacia un camino costero, cubriéndolo, con nebkas con un frente elevado y una cola en la que se han desarrollado ripple marks (Fig. 10 A,B,C). Otras evidencias de alimentación reciente en la zona distal son los frentes transgresivos de este amplio cordón de dunas anteriores preexistente, con aporte masivo de arenas hacia línea férrea, que se localiza en la zona interdunaria que es casi inexistente (Fig. 10 D). Hacia barlovento, las dunas ya estabilizadas por un denso bosque, muestran evidencias de un menor e incipiente soterramiento por arenas.

\section{Discusión y conclusiones}

\section{Cambios en el uso de suelo del valle del Aconcagua}

Los impactos causados por los cambios de uso del suelo por la agricultura, la industria, la forestación y el crecimiento urbano, han sido globalmente identificados, desde la modificación de la calidad del agua, pérdida de biodiversidad, e inestabilidad de los ecosistemas (Koning et al., 1998), como la modificación de los procesos hidrológicos en cuencas (Iroume, 1997) o bien, en el impacto en el escurrimiento superficial debido a la expansión urbana (Henríquez et al., 2006).

Figura $\mathrm{N}^{\circ} 9$

Microbarján, en cordón litoral desmantelado por el oleaje

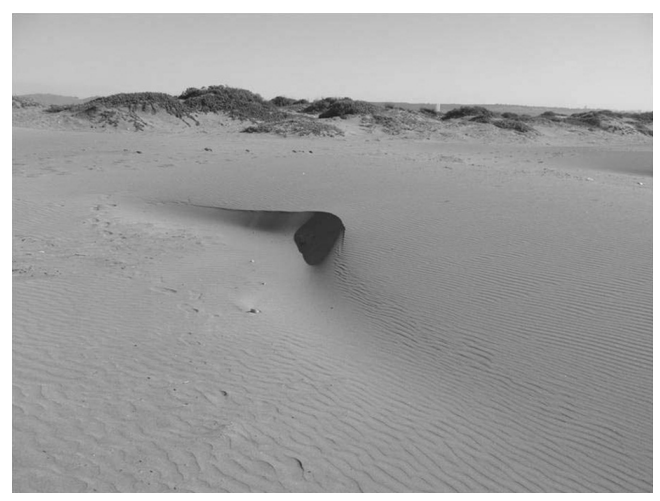

Fuente: Colección personal de los autores, noviembre de 2009. 
Figura $N^{\circ} 10$

Dunas primarias, Ritoque

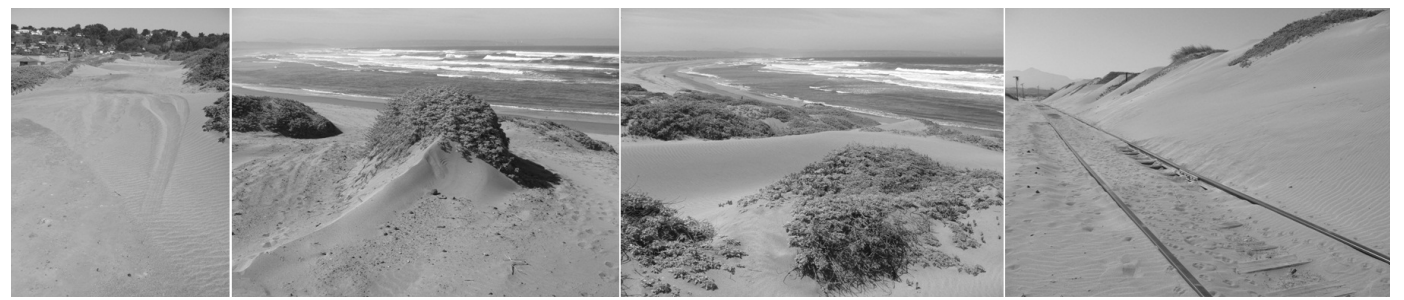

A y B: nebkas recientes desarrolladas sobre un camino. C: cordón litoral, desarrollo de nebkas y dunas embrionarias en playa con perfil erosional. D: frente transgresivo en ambiente de depresión interdunaria.

Fuente: Colección personal de los autores, noviembre de 2009.

La expansión territorial de la actividad frutícola en el valle es un hecho territorialmente comprobado (Castro et al., 2010, Meza 2010; Soto et al., 2010; Aliaga y Leyton 2008), siendo un proceso en constante crecimiento, que traspasa el límite del suelo agrícola de valle y penetra en terrenos con pendientes más allá del umbral morfodinámico aconsejable para la explotación agrícola sustentable.

Además de las modificaciones topográficas necesarias de realizar para las labores agrícolas de frutales, se han identificado los impactos de estas actividades en terrenos con pendiente, como conos y laderas. Así, en ambientes mediterráneos de Europa, la generación y avance de procesos erosivos del suelo y la vulnerabilidad a las remociones en masa vinculadas a tales acciones y actividades económicas, han sido claramente demostradas y cartografiadas (Rofolfi, 2008; Pelacani et al., 2006a, 2006b; Rodolfi, 2006, Rodolfi y Sanchi 2002).

En Chile, el estudio de las nuevas fronteras agrícolas en el semiárido data de la década de los 80, pero han sido los trabajos más recientes los que han contribuido a establecer las relaciones entre cambio de uso del suelo y sus impactos en el sistema natural y la generación de procesos sinérgicos, tales como el aumento de la amenaza natural y vulnerabilidad (Castro et al., 2009) y pérdida de servicios ambientales (Romero et al., 2004).

En el contexto de los impactos observados en la cuenca vinculados a los cultivos en pendientes, al aplicar la metodología de las ERUs (Modelo de Evaluación Cualitativa de la erosión, de Maerker et al., 2001), Aliaga y Leyton (2008) y Castro et al., 2010) se ha establecido una relación entre los grados de susceptibilidad a la erosión y la posición morfoclimática en el valle, de tal manera que la intensidad de la erosión está asociada principalmente a la condición topográfica del terreno, con una relación directa y significativa con la pendiente (Meza, 2010).

Finalmente, es importante destacar que la tendencia de avance en terrenos de fuerte pendiente continuará, debido a las ventajas comparativas que estas zonas ofrecen. En este sentido, Aliaga y Leyton (2008) calculó que los cultivos en laderas son una alternativa más rentable en la actualidad, a pesar de la mayor inversión inicial en habilitación, debido a la oferta de suelo, su bajo valor y las condiciones favorables frente a factores climáticos adversos, como las heladas.

No obstante todo lo anterior, a escala de sistemas y de subsistemas, el análisis de los usos del suelo y cambios de cobertura vegetacional en el área costera adyacente, no se ve reflejada la relación esperada y planteada en la hipótesis. Por el contrario, el subsistema dunario del Aconcagua, demuestra un aumento de la cobertura vegetacional.

$\mathrm{Al}$ respecto se puede sostener que este incremento se debe a las labores de manejo del campo de dunas, con especies vegetales diversas y a su posición morfoclimática que ha permitido la mantención de esta vegeta- 
ción. La escasa progradación observada en el litoral arenoso se puede atribuir al aumento del aporte sedimentario de la cuenca del Aconcagua, según lo observado en el sistema de desembocadura.

\section{El litoral arenoso}

El litoral arenoso asociado a la cuenca del Aconcagua, visto como un sistema complejo de formas depositacionales vinculadas a la transferencia de masa desde la cuenca, presenta condiciones dinámicas y geoformas de carácter general que dificultan su interpretación en función de la hipótesis planteada. En este contexto, y de acuerdo a los estudios de formas y evolución realizado por Castro (1987), y aplicando los conceptos de transmudación de dunas de Araya-Vergara 1987), se observa que el sistema no ha sufrido avance dunario, y el continuum dunar se aprecia sin cambios evolutivos, lo que podría indicar que no existe transmutación y que el sistema no ha estado en las últimas décadas recibiendo aportes adicionales de sedimentos desde la cuenca.

Tal situación sería difícil de explicar en virtud de los significativos cambios descritos para la cuenca en las últimas décadas y lo observado en las dunas de Copiapó (Paskoff et al., 2003; Arriagada 2009, Soto et al., 2010) y Huentelauquén (cuenca del Choapa, en Arriagada, 2009), asociados a cuencas de dominio semiárido, andinas, con procesos de ocupación del suelo comparables con el Aconcagua.

En este escenario ha sido posible establecer evidencias de cambio en la desembocadura, en la playa arenosa y en las dunas, a través de la búsqueda de aquellas formas y subsistemas que han sufrido los procesos de transmutación. Este proceso es de poco alcance espacial pero muy significativo en términos de su interpretación dinámica. Los cambios observados en la playa dan cuenta de la transmutación de dunas primarias de nebkas a barjanes, cambio que implica un mayor aporte de sedimentos reciente a la línea de costa, puesto que ese sector históricamente ha estado cubierto por dunas tipo nebkas. El otro cambio en las dunas es la modificación de barjanoides a blowout, que implica un proceso inverso al ya se- ñalado. Ello ocurre en la zona media de la ensenada en el área de desembocadura del estero Mantagua, en donde la playa y el cordón de dunas anteriores están recibiendo más arenas, pero estas no son transferidas hacia el interior, y los barjanoides se están convirtiendo en blowout y podrían derivar a parabólicas. Es decir, la deflación en las dunas barjanoides ha generado una transmutación a blowout (erosión), deflacionando las arenas hacia frentes transgresivos generando transmudación. Ello concuerda con un litoral cercano, que presenta una zona de rompiente más estrecha que en la parte distal, del tipo intermedia, con tendencia a patrones longitudinales, que contribuye más al transporte en dirección de la deriva que de forma transversal.

En la parte distal, la evolución de dunas ha seguido su tendencia evolutiva en el contexto de las dunas longitudinales, pero con evidencias de aporte de masa, en la medida que existe un cordón de dunas anteriores en desarrollo y avances hacia el interior del campo. El litoral cercano, muy ancho, intermedio, pero de tendencia transversal, da cuenta de cómo se realiza el transporte y transferencia de sedimentos desde la cuenca a la zona distal. Los patrones observados coinciden con el modelo de Araya-Vergara (1996), que indica que en las partes distales de las ensenadas se localizan los mayores anchos de rompientes y de dunas, situación también verificada por Soto (2003) y Soto y Arriagada (2007) en ensenadas de Chile central.

En relación al sistema de desembocadura, no se observan evidencias de transformaciones importantes en sus formas esenciales, y solo se aprecian cambios morfológicos en la flecha litoral, asociados a la influencia fluvial y del oleaje tal como lo señaló Cortez (2002), pero que además, está sometido a fuertes fluctuaciones estacionales, tal como demostrado por la playa y los bancos efímeros de rodados. Esta situación estacional a su vez, concuerda con lo observado por Martínez (2007) quien identificó importantes variaciones de la línea litoral, estableciendo oscilaciones de $-368 \mathrm{~m}$ y $+123,8 \mathrm{~m}$, en el período 1945 y 2006, pero discutiendo tales resultados en el contexto de zonas costeras fuertemente urbanizadas. 
En consecuencia con lo anteriormente expuesto, es posible establecer que las relaciones existentes entre los cambios de uso del suelo intensivos que se están desarrollando en la cuenca están siendo reflejados en línea costera, sobre todo en la parte media y distal de la ensenada que ha derivado en la evolución transmutativa de dunas en el primer caso y en la acreción de las formas de dunas anteriores y transgresión de frentes dunarios en el campo de dunas de Ritoque.

De la misma manera resalta la aseveración de Wang et al. (2010) quien señala que los cambios climáticos y de uso del suelo en las cuencas hidrográficas (río Amarillo) están fuertemente relacionados con los cambios en la dinámica sedimentaria de la zona estuarial y de la desembocadura, ilustrando así la influencia del Antropoceno en la zona de interacción entre cuenca y el litoral.

Si bien las evidencias son más bien de carácter local, y a través de evidencias morfológicas específicas, el tipo de modificación observada y las condiciones de dinámica productiva de la cuenca y de geodinámica del litoral, permite concluir que hay aumento de masa desde la cuenca hacia su litoral correlativo, constatando así de manera relativa la hipótesis planteada.

En este contexto y en función de los escenarios futuros de cambio económico y ambiental, se plantea la necesidad de realizar estudios de mayor profundidad y crear las metodologías apropiadas para poder establecer los procesos interactivos entre cuenca y litoral.

A manera de poder concluir respecto a la tendencia evolutiva, las transformaciones de la cuenca en el escenario del cambio climático, tendería al aumento de la erosión y consecuente carga de masa sedimentaria al sistema hidrológico, que debiera verse reflejado, no necesariamente en una acreción de la línea litoral, sino más bien, en transformaciones en las formas de las dunas y los límites del sistema dunar.

\section{Referencias bibliográficas}

ALIAGA C. y H. LEYTON. Evaluación de la pérdida de suelo y de su calidad, asociado al proceso de expansión urbana y reconversión productiva, Valle del Aconcagua, Comunas de Los Andes, Quillota y Concón. Memoria para optar al título de Ingeniero Civil en Geografía de la Universidad de Santiago de Chile, Facultad de Ingeniería, 2007.

ARAYA-VERGARA, J. F. Significance of barchans in Beach-dune Systems interactions in Central Chile. Thalassas, 1986, vol. 4, № 1, p. 23-27.

ARAYA VERGARA, J. F. The evolution of modern coastal dune systems in central Chile. International Geomorphology 1986 Part II. Ed. V. Gardiner. John Wiley \& Sons Ltd., 1987, 1231-1244.

ARRIAGADA, J. Geomorfología estuarial comparada en la zona semiárida de Chile. Casos de Copiapó y Choapa. Tesis para optar al grado de Magíster en Geografía, Mención Recursos Territoriales. Universidad de Chile, Facultad de Arquitectura y Urbanismo, 2009.

BACHMANN, P.; DELGADO, L. y MARÍN, V. Hacia un manejo ecológico integral de zonas costeras en Chile: Contribuciones del proyecto Ecomanage. Santiago: Ed. Bachmann, Delgado y Marín. 2007.

CASTRO C. Transformaciones geomorfológicas recientes y degradación de las dunas de Ritoque. Revista de Geografía Norte Grande, 1987, № 14, p. 3-13.

CASTRO, C. P.; SOTO, M. V.; FERNÁNDEZ, R.; MARKER, M. y RODOLFI, G. Impacto en la geodinámica actual del valle de Nantoco, cuenca del río Copiapó, asociado a la reconversión productiva. Revista de Geografía Norte Grande, 2009, № 42, p. 81-99.

CASTRO, C. P. \& ARRIAGADA, J. En búsqueda de un reclasificación de los sistemas actuariales de Chile semiárido, comprendido entre los ríos Copiapó y Aconcagua. En: Porto Alegre, Workshop Geologia Marinha, Universidade Federal do Rio Grande do Sul. Instituto de Geociências, Centro de Estudos de Geologia Costeira e Oceânica, 2009. 
CASTRO C. P.; MARKER, M. y C. ALIAGA. Evaluación de la pérdida de suelo, asociada al proceso de expansión urbana y reconversión productiva. Caso: Comunas de los Andes, Quillota y Concón, Valle del Aconcagua. Revista de Geografía Norte Grande, 2010, No 45, p. 41-49.

CORTEZ, C. Observaciones dinámicas y geomorfológicas en el estuario del Aconcagua, Chile central. Memoria para optar al título de Geógrafo. Universidad de Chile, Chile, 2002.

COMISIÓN EUROPEA. Hacia una estrategia europea para la gestión integrada de las zonas costeras. Principios generales y opciones políticas. Luxemburgo: Ed. Oficina de publicaciones oficiales de las comunidades europeas, 1999.

FERNÁNDEZ R. Evaluación espacial de procesos erosivos y su influencia sobre el corredor de comercio Las Leñas. VI Región del Libertador Gral. Bernardo O'Higgins. Memoria para optar al título de Geógrafo. Universidad de Chile, Chile, 2006.

GARREAUX, R.; ACEITUNO, P.; MUÑOZ, R.; ROJAS, M. y MONTECINOS, A. El clima de Chile está cambiando. Comunicación del Proyecto ACT-19. Variabilidad climática en Chile: evaluación, interpretación y proyecciones. Santiago: DGF, Universidad de Chile, 2008.

HENRÍQUEZ, C.; AZÓCAR, G. y M. AGUAYO. Cambio de uso del suelo y escorrentía superficial: aplicación de un modelo de simulación espacial en Los Ángeles, VIII Región de Biobío, Chile. Revista de Geografia Norte Grande, 2006, №36, p. 61-74.

IROUME, A. Estudio de los procesos hidrológicos en una cuenca experimental forestal andina de la IX Región, Chile. Bosque, 1997, vol. 18, No 1, p. 73-81.

RODOLFI, G. \& ZANCHI, C. Cultures viticoles et phénomenes d'instabilité géomorpholique en Italie centrale. Paris: Reveue officielle de I'Union Francaises des Geologues, 2002.
RODOLFI, G. Erosione hídrica del suolo in ambiente mediterraneo: valutazione diretta e indirecta in aree Campione e Bacini idrografici. Genova: Ministero dell Universita e Della Ricerca Progetti di Rilevante Interesse Nazionale, 2006.

MÄRKER M.; MORETTI S. \& RODOLFI G. Integrated water resources management system. Capítulo "assessment of water erosion processes and dynamics in semi-arid regions of southern africa (kwazulu / natal, rsa, and swaziland) using the erosion response units concept (eru)". Revista geogr. fis. dinam. quat., 2001, vol. 24, issue 1.

MARTíNEZ, C. Cambios en la línea litoral de las bahías de Algarrobo y Concón, Chile central, a través de un modelo de ajuste. Investig. mar., 2007, vol. 35, № 2, p. 99-112.

MARTÍNEZ, C. y CORTEZ, C. Características hidrográficas y sedimentológicas en el estuario del río Aconcagua, Chile central. Revista de Geografía Norte Grande, 2007, N³7, p. 63-74.

MASSELINK, G.; KROON, A. \& DAVIDSON-ARNOTT, R. Morphodynamics of intertidal bars in wave-dominated coastal settings-a review. Geomorphology, 2006, № 73, p. 33-49.

MEZA, M. Evaluación del recurso suelo como indicador de degradación ambiental por uso y manejo en el contexto de la reconversión productiva, cuenca de Quillota, curso medio del río Aconcagua. Tesis para optar al grado de Magíster en Geografía. Universidad de Chile, Chile, 2010.

PASKOFF, R. R e che r ches geomorphologiques dans le Chili Semi-aride. Paris: Bordeaux, 1970.

PASKOFF, R.; CUITIÑO, L. y MANRÍQUEZ, H. Origen de las arenas dunares de la Región de Copiapó, Desierto de Atacama, Chile. Revista Geológica de Chile, 2003, vol. 30, №2, p. 355-361.

PELACANI, S.; UNGARO, F.; LOMBARDI, L. \& RODOLFI, G. Applicazione di techiche 
geostatistiche e GIS per la caratterizzazione dei suoli e della loro erodabilita. In: RODOLFI, G. Water erosion in mediterranean environment: direct and indirect assessment in test areas and catchments. Genova: Ministero dell Università e Della Ricerca Progetti di Rilevante Interesse Nazionale, 2006a.

PELACANI, S.; LOMBARDI, L.; MAERKER, M. \& RODOLFI, G. Caratterizzazione dei processi geomorfici di versante nel bacino del torrente orme (Toscana Centrale) sulla base delle unita di risposta erosiva - ERU. Genova: Ministero dell Università e Della Ricerca Progetti di Rilevante Interesse Nazionale, 2006b.

PANDA, D. K. y MOHANTHY, A. K. S. Recent trends in desiment load of tropical (Peninsular) river basin of India. Global and Planetary Change, 2011, № 75, p. 108-118.

RESTREPO, J. D.; J ZAPATA, P.; DÍAZ, J. M.; GARZON-FERREIRA, J. \& GARCÍA, C. B. Fluvial fluxes into the Caribbean Sea and their impact on coastal ecosystems: The Magdalena River, Colombia. Global and Planetary Change, 2006, N 50, p. 33-49.

ROMAY, D. y MACEIRA, A. La gestión integrada de las zonas costeras y de la Directiva Marco del agua. El caso de Galicia. In simposio Internacional La nueva política del agua: Barcelona, 2006.

ROMERO, H. y VÁSQUEZ, A. Evaluación ambiental del proceso de urbanización de las cuencas del piedemonte andino de Santiago de Chile. EURE, 2005, vol. XXXI, No 94, p. 97-118.

SANTSCHI, P. H.; OKTAY, S. D. \& CIFUENTES L. Carbon Isotopes and iodine concentrations in a Mississippi River delta core recording land use, sediment transport, and dam building in the river's drainage basin. Marine Environmental Research, 2007, No63, p. 278-290.
SHORT, A. D. Waves-dominated beachs, In: SHORT A (ed). Handbook of beach and shoreface morphodynamics. Chichester: John Wiley \& Sons, 1999, p. 173-191.

SLATTERY, M. C. \& PHILLIPS, J. D. Control on sediment delivery in coastal plain rivers. Journal of environmental Management, 2011, No 92, p. 284-289.

SOTO, M. V. Análisis de los cambios asociados al oleaje en una ensenada de la zona central de Chile. Anales de la Sociedad Chilena de Ciencias Geográficas, 2003, p. 135-144.

SOTO, M. V. y ARRIAGADA, J. Características dinámicas de ensenadas estructurales en Chile central. Maitencillo y Papudo, Región de Valparaíso. Revista de Geografía Norte Grande, 2007, №38, p. 99-112.

SOTO, M. V.; CASTRO, C. P. y MARCILLO, J. L. Cambios de uso del suelo en la cuenca del Aconcagua y su impacto en el litoral arenoso correlativo de Ritoque. Región de Valparaíso. Talca: Congreso Nacional de Geografía, 2009.

SOTO. M. V.; MÄRKER, M.; ARRIAGADA, J.; CASTRO, C. y RODOLFI, G. Evaluación de la amenaza natural en ambiente semiárido, sustentada en la geomorfología y el modelamiento de índices topográficos. Salamanca Región de Coquimbo, Chile. Investigaciones Geográficas, 2010, № 42, p. 37-56.

VERSTAPPEN, H. On dune types, familias and sequences in areas of unidirectional winds. Gottiner Geogr. Abh, 1972, No 60, p. 341-353.

WANG, H.; BI, N.; SAITO, Y.; WANG, Y.; SUN, X.; ZHANG, J. \& YANG, Z. Recent changes in sediment delivery by the Huanghe (Yellow River) to the sea: Causes and environmental implications in its estuary. Journal of Hydrology, 2010, №391, p. 302-313. 